\title{
CAPACIDAD INNOVADORA: CÓMO FOMENTARLA, SEGÚN DOCENTES DE CIENCIAS ECONÓMICAS E INGENIERÍA DE LA UMNG
}

\section{INNOVATIVE CAPACITY: HOW TO PROMOTE IT. BY TEACHERS OF ECONOMICS AND ENGINEERING OF UMNG UNIVERSITY}

\author{
Jhon Alexander Valdelamar-Zapata ${ }^{1}$ \\ Yury Leandra Ramírez-Cruz \\ Paula Daniela Rodríguez-Rivera ${ }^{3}$ \\ María Eugenia Morales-Rubiano ${ }^{4}$
}

Recibido: abril 14 de 2015 Aceptado: junio 30 de 2015

\section{Resumen}

Actualmente los docentes universitarios desempeñan un papel importante en la generación de capacidades innovadoras en sus estudiantes, ya que son el eje transmisor de dicho conocimiento. En este sentido, se busca identificar las concepciones sobre innovación que tienen los docentes de planta, de las facultades de Ciencias Económicas e Ingeniería de la Universidad Militar Nueva Granada (UMNG) y cómo éstas inciden en la forma como los docentes fomentan procesos innovadores en los estudiantes. Se realizó una búsqueda de información en bases de datos científicas indexadas, sobre los temas de innovación y enseñanza desde el punto de vista del docente. Luego se hicieron encuestas de manera virtual y presencial a la población objeto de estudio, compuesta por 131 docentes. Se encontraron pequeñas diferencias de pensamiento respecto a las concepciones y metodologías que tienen los docentes de las facultades mencionadas, evidenciando al final, que existe una relación directa entre la trayectoria laboral y las nociones que han desarrollado respecto a la innovación. Se concluye que los docentes, con base en su experiencia profesional y laboral, consideran entre las mejores metodologías para fomentar la innovación: la realización de proyectos, estudios de caso, el contacto con empresarios innovadores y la investigación de experiencias.

Palabras clave: innovación, concepciones docentes, fomento de la innovación, instituciones de educación superior.

\begin{abstract}
Nowadays university teachers play an important role generating innovative skills in their students, as they are the promoters of such knowledge. In this sense, it seeks to identify the conceptions on innovation that full time teachers of Economics and Engineering faculties of the Universidad Militar Nueva Granada (UMNG) have, and how these affect the way teachers encourage innovative processes in students. An information search was performed in indexed scientific databases on the topics of innovation and education from the point of view of the teachers. Later, virtual and face surveys were done to a total of 131 teachers of the study population. Small differences in thinking about the concepts and methodologies of the teachers of the faculties mentioned above were found, showing at the end, that there is a direct relationship between the labor paths and notions that they have developed regarding innovation. It is concluded that teachers based on their professional and work experience, considered among the best methodologies to promote innovation: the implementation of projects, case studies, being in contact with innovative entrepreneurs, and research experiences.
\end{abstract}

Keywords: Innovation, teachers' conceptions, fostering innovation, institutions of higher education.

\footnotetext{
1 Estudiante de Administración de Empresas, Universidad Militar Nueva Granada, Colombia. e-mail: u2203776@unimilitar.edu.co

2 Estudiante de Contaduría Pública, Universidad Militar Nueva Granada, Colombia. E-mail: u2302748@unimilitar.edu.co

3 Estudiante de Contaduría Pública, Universidad Militar Nueva Granada, Colombia. E-mail: u2302755@unimilitar.edu.co

4 Docente Facultad de Ciencias Económicas, Universidad Militar Nueva Granada, Colombia.

E-mail: maria.morales@unimilitar.edu.co
} 


\section{Introducción}

Actualmente se vive en un entorno de globalización y alta competencia, donde la innovación se convierte en un elemento estratégico de competitividad y diferenciación para las organizaciones. En este sentido, las universidades juegan un rol crucial dentro del contexto social y económico, como productoras y transmisoras de conocimiento al entorno (Lee, 1995; Leydesdorff \& Etzkowitz, 1996; D'Este \& Patel, 2007). Parte de esta responsabilidad recae en los docentes, que en su labor de enseñanza-aprendizaje transfieren, no solo sus conocimientos, sino las concepciones que han adquirido a lo largo de su trayectoria laboral y experiencia profesional contribuyendo al desarrollo de procesos creativos en sus estudiantes, base fundamental para la innovación. Es importante considerar que las concepciones sobre innovación que tienen los docentes, se transfieren al estudiante de manera que pueden incidir en aspectos diferenciadores del perfil profesional, ya que la comprensión de las dinámicas asociadas a este fenómeno, les puede proporcionar a los estudiantes una ventaja en el mundo laboral.

El presente artículo tiene como objetivo identificar las concepciones sobre innovación que tienen los docentes de planta de las facultades de Ciencias Económicas e Ingeniería de la Universidad Militar Nueva Granada, UMNG, y cómo éstas inciden en la forma como los docentes fomentan procesos innovadores en los estudiantes. La razón por la cual se decidió indagar en las facultades anteriormente mencionadas radica en que hoy en día los profesionales egresados de éstas, son los más demandados por el mercado laboral colombiano (El Espectador, 2014), y adicionalmente porque inciden directamente en los procesos de innovación que se pueden llevar a cabo en una organización.

El trabajo se enfoca en los factores que influyen en la experiencia tanto profesional como laboral que han tenido los docentes de las facultades referidas con entidades de ciencia, tecnología e innovación, y el impacto que se debe alcanzar con la misma; en este sentido el artículo busca identificar cuáles son las metodologías que los docentes consideran apropiadas con el fin de generar, fortalecer e incentivar las capacidades para la innovación en sus estudiantes.

Para dar cumplimiento al objetivo de este artículo, se desarrolló un marco teórico el cual se compone de una revisión bibliográfica acerca de algunas definiciones de innovación y la influencia de la trayectoria laboral y profesional de los docentes en sus concepciones, identificando la importancia de los factores mencionados. Luego se presentan los resultados que se obtuvieron de la encuesta aplicada, tomando principalmente los factores de: trayectoria en instituciones del Sistema Nacional de Ciencia, Tecnología e Innovación, concepciones, y metodologías para la enseñanza de la innovación; finalmente, a partir de lo indagado se establecen las conclusiones.

\section{Marco teórico y metodología}

\subsection{Definiciones sobre innovación}

Es común que en la sociedad actual, cuando algo deja de funcionar y así mismo deja de dar los beneficios que se esperaban y no suple las necesidades para lo cual se creó, sea momento de cambiarlo o modificarlo para que se pueda superar dicha situación (Silva, 2013). Lo anterior hace pensar que la innovación nace de una necesidad inherente de la sociedad al cambio, pero a ciencia cierta ¿qué es la innovación?, el manual de Oslo define innovación como:

"La introducción de un nuevo o significativamente mejorado, producto (bien o servicio), de un proceso, de un nuevo método de comercialización o de un nuevo método organizativo, en las prácticas internas de la empresa, la organización del lugar de trabajo o las relaciones externas" (OCDE, 2006, p.56). 
Por otra parte Nicholls (1983, citado en Marcelo, 2013 , p. 38) define innovación como "una idea, objeto, o práctica percibida como nueva por un individuo o individuos, que intenta introducir mejoras en relación a objetivos deseados, que por naturaleza tiene una fundamentación, y que se planifica y delibera". Como se puede apreciar en las anteriores definiciones está inmersa la necesidad de cambio en el producto, proceso, organización y mercadotecnia (OCDE, 2006).

\subsection{Factores que pueden determinar las con- cepciones docentes}

¿Qué concepciones tienen los docentes acerca de innovación? Antes de responder esta pregunta es importante considerar que las concepciones de los docentes están ligadas a sus atribuciones, metas, experiencias previas, así como sus complejos procesos motivacionales (Díaz-Barriga, 2010). Esto implicaría conocer el perfil y la trayectoria del docente, con el fin de identificar cómo afectan esos aspectos la forma en que los docentes conciben la innovación y se aproximan al proceso de trasmisión de conocimiento, siendo este el primer factor a considerar dentro del presente estudio.

Entre otros factores que se relacionan con las creencias y actitudes de los docentes, Díaz-Barriga (2010) menciona: la metodología de enseñanza de la innovación, su percepción al respecto y las definiciones de la misma. En éste sentido, se encontró que los docentes consideran que la innovación debe estar acompañada de la tecnología, instrucciones respecto a la misma y más interacciones con ella. Sin embargo, se reconoce que hay dificultades para innovar aun cuando ésta se implemente (Davis, Hartshorne, \& Ring, 2010). Por lo tanto es necesario considerar la tendencia por parte de los docentes al referirse a la innovación desde un punto de vista tecnológico, concepción que se queda corta dada la multiplicidad de innovaciones que se pueden dar, tal como lo menciona la OCDE, porque innovar es un proceso más exigente en un mundo de organizaciones competitivas ávidas de factores diferentes.

\subsection{El perfil docente y la capacidad innovadora}

Como se ha mencionado, existen diversos conceptos de innovación y su aplicación suele darse básicamente en ámbitos económicos y empresariales, sin embargo en los últimos años según Silva, $(2013$, p. 14) "las reflexiones sobre la innovación en el campo educativo han venido incrementándose". Es así como Adell (1997 citado en Rodríguez, 2012, p.1538) menciona un nuevo perfil del docente, donde su misión debe ser la de facilitador, guía, consejero y creador de hábitos y destrezas al buscar, seleccionar y darle tratamiento a la información hallada en fuentes idóneas.

Por lo anterior, para que los docentes sean facilitadores del conocimiento es necesario considerar los aspectos que Richardson (1996, citado en Marcelo, 2013, p.30) menciona de una forma comprensible y directa: "En primer lugar las creencias influyen en la forma como aprenden los profesores; y en segundo lugar las creencias influyen en los procesos de cambio que los profesores puedan protagonizar".

Cuando el docente no facilita el conocimiento adecuado a los estudiantes, todo se torna en un proceso de aprendizaje dedicado a una transferencia unilateral de contenidos, que se aleja del resultado buscado, tal como lo menciona Hernández (2010, p.4) "el proceso de aprender es un proceso activo en el que se producen cambios en el cerebro del aprendiz, ya que manejar los contenidos no es simplemente transportarlos sino manipularlos y darles profundidad y significado".

\subsection{Metodología}

La investigación que soporta este artículo, se basó en un enfoque cuantitativo-cualitativo, de tipo exploratorio, tomando como técnica de recolección de información la encuesta. En función del objetivo formulado, la metodología se desarrolló en cuatro fases: en primer lugar se realizó una búsqueda de información en bases de datos científicas referente a los temas de innovación y ense- 
ñanza desde el punto de vista del docente como facilitador del conocimiento en un entorno educativo y social. Posteriormente se procedió con la recolección de datos, a través de una encuesta que abordó tres dimensiones importantes: i) Las concepciones que se tienen sobre la innovación, ii) EI proceso de acercamiento que tienen los docentes sobre la temática, y iii) La manera como ellos perciben y seleccionan aquellos elementos que consideran importantes promover en el proceso del ejercicio docente.

El instrumento de recolección de información se aplicó en dos etapas: en la primera, se envió de manera virtual a cada uno de los 131 docentes de la población objetivo, docentes de planta de las facultades de Ciencias Económicas e Ingeniería de la UMNG, con el fin de facilitar la recopilación de datos, obteniendo la respuesta de 32 docentes, 16 de cada facultad que integran los programas de Ingeniería Civil, Industrial, Mecatrónica, Multimedia y Telecomunicaciones en el caso de la Facultad de Ingeniería y de Administración de Empresas, Contaduría Pública y Economía en el caso de la Facultad de Ciencias Económicas, como se presenta en la tabla 1.

\begin{tabular}{|l|l|}
\hline $\begin{array}{l}\text { Porcentaje de error } \\
\text { aceptado (e) }\end{array}$ & $5 \%$ \\
\hline $\begin{array}{l}\text { Nivel de confianza } \\
\mathbf{9 0 \%}(\mathbf{Z})\end{array}$ & 1.64 \\
\hline $\begin{array}{l}\text { Desviación estándar } \\
\mathbf{( )}\end{array}$ & 0.5 \\
\hline $\begin{array}{l}\text { Tamaño de la pobla- } \\
\text { ción (N) }\end{array}$ & $\begin{array}{l}131 \text { Docentes de planta de } \\
\text { las Facultades de Ciencias } \\
\text { Económicas e Ingeniería }\end{array}$ \\
\hline Total muestra & 89 \\
\hline
\end{tabular}

Tabla 1. Determinación de la muestra y margen de error

En la segunda etapa, se procedió con la aplicación del instrumento a la población restante de docentes de manera presencial. Al finalizar las etapas anteriores, se llegó a un total de 89 docentes $(68 \%$ de la población objetivo), de los cuales 41 (46\% de la muestra) corresponden a la Facultad de Ciencias Económicas y 48 (54\% de la muestra) a la Facultad de Ingeniería.

Al momento de analizar los resultados, con el fin de dar cumplimiento al objetivo principal, se tomaron en cuenta aspectos clave de los siguientes factores: trayectoria, concepciones y definiciones y el método de enseñanza.

\section{Resultados y discusión}

Se encontró que en la muestra, 67 docentes son hombres con un promedio de edad de 47 años y 22 docentes son mujeres con un promedio de edad de 44 años. Teniendo en cuenta que las concepciones de los docentes están ligadas a sus atribuciones, metas y experiencias (Díaz-Barriga, 2010), este resultado juega un papel importante dado que la edad influye en los aspectos anteriormente mencionados.

Adicionalmente se encontró que el $87.64 \%$ de los docentes se han desempeñado en el sector educativo dado que se relaciona directamente con el oficio de la docencia. Sin embargo, no ha sido el único sector donde ellos han ejercido su profesión, se encontró que tienen experiencia especialmente en los sectores de servicios, industrial y comercio, entre otros.

Teniendo en cuenta el contexto actual de globalización y alta competencia, donde la innovación se convierte en un elemento estratégico de competitividad y diferenciación para las organizaciones (Lee, 1995; Leydesdorff \& Etzkowitz, 1996; D'Este \& Patel, 2007; Morales, Sanabria, \& Plata, 2014); los docentes son pieza fundamental para que los profesionales potencialicen su capacidad de innovar. No obstante, se evidenció en los resultados, que el $57 \%$ de los docentes encuestados no han participado en proyectos de innovación, lo cual reduce su capacidad de aumentar las competencias de los estudiantes en este aspecto, si es que se van a basar en este tipo de experiencias. 
Sin embargo, participar en proyectos de innovación no es la única alternativa para estar al tanto sobre este tema, ya que también se puede tener conocimiento al respecto a través de la interacción con las instituciones que hagan parte del Sistema Nacional de Ciencia, Tecnología e Innovación, SNCTI, que tienen como objetivo "crear sinergias e interacciones para que Colombia cuente con una cultura científica, tecnológica e innovadora" (Colciencias, 2014). Dicho sistema está compuesto por instituciones tecnológicas, productivas, científicas, de apoyo, de apoyo regional y entidades financieras. Bajo este orden de ideas, se encontró que los docentes, en su mayoría han tenido relación laboral con las instituciones científicas y productivas, especialmente los docentes de ingeniería, y los docentes de ciencias económicas respectivamente.

En cuanto a las concepciones y definiciones de innovación, se encontró que los docentes de la Facultad de Ingeniería asocian "innovación" en su mayoría con competitividad, conocimiento, novedad tecnológica y nuevos desarrollos; mientras que los de la Facultad de Ciencias Económicas lo hacen con cambio, competitividad, novedad tecnológica y nuevos desarrollos. Existe una diferencia entre ambas concepciones en el sentido de los términos de conocimiento y cambio, el cual se puede entender porque las ingenierías se sustentan en ciencias como la física, química y matemáticas, entre otras (CIARP, 2014) y por ende es de vital importancia tener conocimiento si se quiere innovar; mientras que las Ciencias Económicas, se fundamentan en las ciencias sociales que buscan generar bienestar, por tal motivo innovar implica un cambio en la sociedad.

Analizando el contexto educativo, los docentes buscan generar interés y fomentar capacidades de innovación en los estudiantes, permitiéndoles potencializar dicho conocimiento y mejorar sus competencias laborales, tal como se evidencia en la figura 1; en ella se muestran las metodologías que los docentes consideran más apropiadas a la hora de generar capacidades innovadoras en los estudiantes.

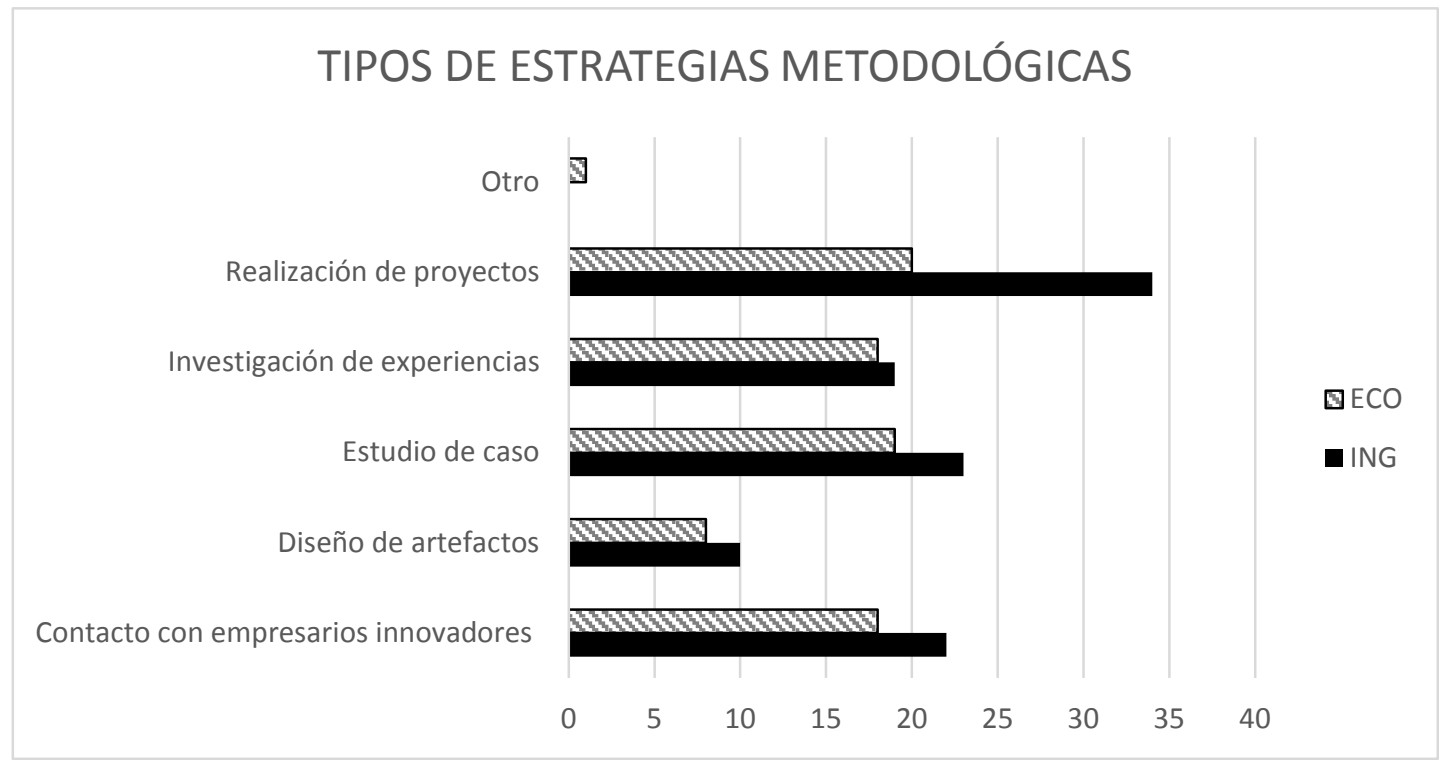

Figura 1. Tipos de estrategias metodológicas 
De la figura 1 se infiere que los docentes de ingeniería consideran la realización de proyectos como la mejor forma para que los estudiantes apropien prácticas que puedan fomentar su capacidad de innovación, y de igual manera lo consideran con estrategias metodológicas basadas en estudios de caso, el contacto con empresarios innovadores y la investigación de experiencias. Por otra parte, los docentes de ciencias económicas comparten las anteriores estrategias, aunque en una proporción un poco inferior respecto a los docentes de ingeniería. Sin embargo la metodología menos utilizada por los docentes de ambas facultades fue el diseño de artefactos, siendo una respuesta poco común para los docentes de ingeniería quienes normalmente suelen estar relacionados con este tipo de metodologías.

Asimismo, en cuanto al mejor momento de introducir al estudiante de pregrado en el contexto de la innovación, en concordancia con las metodologías anteriormente descritas, los docentes consideran que debería ser al inicio de su carrera (58\%), de los cuales el $27 \%$ son de Ciencias Económicas y el 31\% de Ingeniería. También se encontró que los docentes piensan que la mejor forma para desarrollar capacidades innovadoras en los estudiantes es dentro del programa académico, evidenciado en que el $73 \%$ de los docentes están de acuerdo con que se deben incluir estrategias que fomenten la innovación en actividades curriculares, más que actividades extracurriculares.

Finalmente, al indagar por el nivel de competencia que deberían alcanzar los estudiantes frente a procesos de innovación curriculares, los docentes consideran apropiado que se debe: "promover la realización de proyectos para el desarrollo de casos concretos de innovación"; es decir, el grado más alto de exigencia a alcanzar, según lo planteado en el instrumento, pues involucra que el estudiante explote su potencial creativo y de esta forma vaya construyendo y fortaleciendo sus capacidades en el ámbito de la innovación.

\section{Conclusiones}

En primer lugar, algunos factores que pueden determinar las concepciones de los docentes sobre innovación son: la participación en proyectos de innovación, su trayectoria laboral y experiencia profesional. Los resultados muestran que los docentes de Ciencias Económicas son los que tienen mayor relación laboral con instituciones del SNC$\mathrm{Tl}$, concretamente con instituciones productivas, mientras que los docentes de Ingeniería, han hecho lo propio con organizaciones científicas.

En concordancia con lo anterior, y de acuerdo con lo planteado por Díaz-Barriga (2010), respecto a que las concepciones de los docentes se ven influidas por su experiencia, se encontró que los docentes de Ingeniería relacionaron innovación con conocimiento y los de Ciencias Económicas con cambio, a pesar de haber coincidido en otras concepciones relacionadas con términos como competitividad, novedad tecnológica y nuevos desarrollos.

También se observó que los docentes, a fin de generar, fortalecer e incentivar capacidades para la innovación en los estudiantes, buscan utilizar estrategias metodológicas tales como la realización de proyectos, estudios de caso, el contacto con empresarios innovadores y la investigación de experiencias, las cuales sustentadas en su experiencia profesional y laboral, pueden traer a su consideración mejores resultados para apropiar prácticas que los lleven a procesos innovadores. De esta manera se pudo contemplar que los docentes de Ingeniería consideran apropiada la realización de proyectos en mayor proporción que los de Ciencias Económicas.

Finalmente, para complementar dichas capacidades, los docentes de las dos facultades consideran que todas las estrategias metodológicas a desarrollar, se deben llevar a cabo dentro de un ambiente curricular y preferiblemente al inicio de la carrera; de esta manera, el estudiante sentirá una vinculación más fuerte con ellas desde el princi- 
pio, hasta el punto que llegue a una apropiación de dicho conocimiento cuando esté finalizando su formación. No obstante, a pesar de que los docentes tienen a consideración muchas metodologías y su experiencia es importante al momento de tratar de fomentar un pensamiento innovador en sus estudiantes, en la realidad existe un ambiente diferente, dado que no todos los docentes aplican actualmente dichas metodologías, e incluso no hacen parte del programa académico de dichas carreras; por lo tanto continúa abierta la pregunta: Qué tan capacitados estarán los futuros profesionales en el tema de innovación?.

\section{Agradecimientos}

Artículo derivado del PIC ECO 1727 "Concepciones sobre innovación en los docentes de planta de Ciencias Económicas e Ingeniería de la UMNG", financiado por la Vicerrectoría de Investigaciones de la Universidad Militar Nueva Granada, vigencia 2014 y del proyecto ECO 912"Mujeres empresarias en Colombia: prácticas empresariales y procesos de innovación", financiado por COLCIENCIAS y la UMNG, vigencia 2011-2014. Igualmente se agradece a Fabián Arley Ninco Hernández, Paola Andrea Plata Pacheco y Jenny Paola Beltrán, jóvenes investigadores de la Facultad de Ciencias Económicas, por su acompañamiento al desarrollo del proyecto en sus diversas fases. De igual forma a Oscar Andrés Layton González, estudiante de Contaduría Pública de la UMNG, integrante del semillero "Estrategia y gestión" de la Facultad de Ciencias Económicas, por su participación en el PIC ECO 1727.

\section{Referencias}

COLCIENCIAS. (8 de noviembre de 2014). Sobre Colciencias. Recuperado de http://www.colciencias.gov.co/sobre_colciencias.

Colegio de Ingenieros y Agrimensores de Puerto Rico [CIARP]. (2014). ¿Qué es Ingeniería?. Recuperado el 24 de noviembre de 2014 de http://ciapr. org/ciapr.org/?option=com_content\&view=article\&id=153\&ltemid=131\&fontstyle=f-larger
D'Este, P., \& Patel, P. (2007). University-industry linkages in the UK: What are the factors underlying the variety of interactions with industry? Research Policy 36, 1295-1313.

Davis, H., Hartshorne, R., \& Ring, G. (2010). Being an Innovative Teacher: Preservice Teachers. International Journal of Education, 1-28. Recuperado el 1 de noviembre de 2014 de http://www.macrothink. org/journal/index.php/ije/article/view/242

Díaz-Barriga, F. (2010). Los profesores ante las innovaciones curriculares. Revista Iberoamericana de Educación Superior, 1(1), 37-57. Recuperado de http://ries.universia.net/index.php/ries/article/ view/35.

El Espectador (2014, 23 marzo). Estas son las carreras universitarias más solicitadas por las empresas en Colombia. Recuperado el 22 de Noviembre de 2014 de http://www.elespectador.com/noticias/educacion/estas-son-carreras-universitarias-mas-solicitadas-empre-articulo-482544.

Hernández, I. (2010). ¿Qué es aprender?. Innovación y experiencias educativas, 1-9. Recuperado el 8 de noviembre de 2014 de http://www.csi-csif. es/andalucia/modules/mod_ense/revista/pdf/Numero_36/INMACULADA_HERNANDEZ_1.pdf.

Lee, Y. S. (1995). 'Technology transfer' and the research university: a search for the boundaries of university-industry collaboration. Research Policy, 843-863.

Leydesdorff, L., \& Etzkowitz, H. (3-6 January 1996). Emergence of a Triple Helix of University Industry Government Relations. Report of the Conference in Amsterdam. Amsterdam: Recuperado el 15 de agosto de 2014 www.leydesdorff.net/th1a/.

Marcelo, C. (enero-marzo de 2013). Las tecnologías para la innovación y la práctica docente. Revista Brasileira de Educação, 25-47. Recuperado de http:// www.redalyc.org/articulo.oa?id=27525615003 
Morales, M., Sanabria, P., \& Plata, P. (2014). Determinantes de la transferencia de propiedad industrial al sector productivo en universidades públicas colombianas. Cuadernos de Administración, 30, 58-70.

OCDE. (2006). Manual de Oslo: Guía para la recogida e interpretación de datos sobre innovación. Tercera edición. OCDE, Comisión Europea y Eurostat. Recuperado el 3 de septiembre de 2014, de http:// www.uis.unesco.org/Library/Documents/OECDOsloManual05_spa.pdf

Rodríguez, A., \& Alvarado, H. (2008). Claves de la innovación social en América Latina y el Caribe. CEPAL: Santiago de Chile. Recuperado el 25 de noviembre de 2014 de: http://repositorio.cepal.org/ bitstream/handle/11362/2536/S0800540_es.pd$\mathrm{f}$ ?sequence $=1$
Rodríguez, J. (2012). Innovación y Tecnología: necesidad de cambios en los procesos de enseñanza. Vivat Academia, 1520-1544. Recuperado el 20 de agosto de 2014 de http://www.ucm.es/info/vivataca/numeros/n117E/DATOSS.htm

Silva, R. (2013). El reto de innovar en el proceso docente universitario. Uni-pluri/versidad, 13-15. Recuperado el 20 de agosto de 2014, de http:// ezproxy.umng.edu.co:2048/login?url=http:// search.proquest.com/docview/1430959938?accountid=30799

Suarez, M. (2013). Interaprendizaje de probabilidades y estadística inferencial con Excel, Winstats y Graph. Universidad Técnica del Norte: Ibarra- Ecuador. 